\title{
Deletion of SarX decreases biofilm formation of Staphylococcus aureus in a polysaccharide intercellular adhesin (PIA) dependent manner
}

\author{
Zhihao Hao \\ Wenzhou Medical University \\ Yinjuan Guo \\ Shanghai Pulmonary Hospital \\ Lulin Rao \\ Wenzhou Medical University \\ Jingyi Yu \\ Wenzhou Medical University \\ Qing Zhan \\ Nanchang University \\ Yanlei Xu \\ Nanchang University \\ Bingjie Wang \\ Tongji University \\ Xiaocui Wu \\ Shanghai Pulmonary Hospital \\ Fangyou Yu ( $\nabla$ wzjxyfy@163.com ) \\ Shanghai Pulmonary Hospital
}

\section{Research Article}

Keywords: Staphylococcus aureus, ica, biofilm formation, sarX, spa

Posted Date: December 15th, 2020

DOI: https://doi.org/10.21203/rs.3.rs-121814/v1

License: (c) (i) This work is licensed under a Creative Commons Attribution 4.0 International License. Read Full License 


\section{Abstract}

Background: Biofilm formation by Staphylococcus aureus is an important virulence determinant mediated by the polysaccharide intercellular adhere (PIA) encoded by ica operon or by surface and extracellular proteins. Previous studies have shown that $S$. epidermidis SarX protein regulated the transcriptional activity of the agrand ica loci and controled the biofilm phenotype, primarily by regulating icaADBC transcription and PIA production.

Results: In this study, our results indicated that biofilm formation and detachment of $S$. aureus were significantly decreased in the sarX mutant strain. sarX mutant in $S$. aureus biofilm formation was related to the production of PIA and not to that of eDNA. RT-PCR results showed that deletion of sarX was associated with a 1.8-fold reduction in spa transcription, which was complemented by sarX. Expression of Spa protein was decreased in sarX mutant strain.

Conclusions: sarX promoted biofilm production of $S$. aureus may mediated primarily through increasing ica operon expression and PIA production. Deletion of sarX was associated with reduction in spa transcription.

\section{Introduction}

Staphylococcus aureus is a human pathogen responsible for a wide diversity of community- and hospital-acquired infections, including relatively benign and fatal systemic disease (pneumonia, endocarditis, mastitis, osteomyelitis, etc.) [1,2]. S. aureus can develop biofilms on host tissues and medical devices[3], leading to chronic infections, with up to $80 \%$ of chronic bacterial infections associated with biofilms[4]. Biofilms formation during $S$. aureus infection are difficult to treat. $S$. aureus can escape the immune defense of the dormitory and the attack of multiple immune factors by forming the biofilm. The dense biofilm can also prevent or delay the infiltration of antibiotic drugs, leading to bacteria at the bottom of the biofilm to produce drug resistance genes, reducing the sensitivity of bacteria to antibiotic drugs[5]. An evaluation of the biofilm formation process of $S$. aureus will likely contribute to our understanding of the infectious process.

Biofilm development and formation generally involve initial adhesion, proliferation, maturation, and diffusion [6]. Biofilm formation is modulated by transcriptional regulators (SarA, MgrA, and Rbf) and various regulatory systems (agr quorum sensing system) by regulating the production of biofilm formation associated factors (surface proteins, polysaccharide intercellular adhesin (PIA), eDNA, and other extracellular components). PIA, poly-NN-acetylglucosamine, were the most common constituents of staphylococcal biofilm, first found in Staphylococcus epidermidis[7]. The biosynthesis of PIA is mediated by the ica operon, which contains four open reading frames (icaA, icaD, icaB, and icaC) and one regulatory gene $(i c a R)$. ica operon encodes the IcaA protein, which has $\mathrm{N}$-acetamide glucanotransferase activity, which can synthesize UDP-N-acetylglucosamine into an oligomer structure with a chain length of 20 residues. ica $A D B C$ operon was negatively controlled by the IcaR and the teicoplanin-associated locus 
regulator TcaR[8]. The transcription regulator TcaR, a member of the multiple antibiotic resistance regulator (MarR) family, is involved in teicoplanin and methicillin resistance in staphylococci[9]. Members of the SarA protein family in $S$. aureus share homology with each other as well as with the MarR family. SarX, a member of the SarA/MarR family of transcriptional regulators, was first identified in $S$. aureus by Manna and Cheung[10]. Transcription of $\operatorname{sar} X$ in $S$. aureus has been shown to be temporal and expressed maximally in stationary phase. Inactivation of sarX did not affect the expression of regulatory genes in the sarA family or saeRS, but it did have a significant negative effect on the transcription of agr. A study revealed that the $S$. epidermidis SarX protein regulates the transcriptional activity of the agr and ica loci and controls the biofilm phenotype, primarily by regulating icaADBC transcription and PIA production[11].

In addition, the cell wall-anchored proteins that participate in biofilm formation are biofilm-associated protein (Bap), ClfB, FnBPs, SasC, SasG and protein A (Spa)[12]. Spa is ubiquitous in S. aureus and is often used in strain typing on the basis of variation in the DNA sequence encoding the $X$ region[13]. Spa, the first surface protein of $S$. aureus, was identified as a model for the characterization of the covalent anchorage of the LPXTG domain proteins by sortase $A$ to the bacterial cell surface[14].

The purpose of this study was to investigate the impact of a sarX deletion mutation on ica, and PIA and biofilm regulation in $S$. aureus.

\section{Materials And Methods}

\section{Bacterial strains, plasmid, and grow conditions.}

The bacterial strains and plasmids used in this study were listed in Table 1. Staphylococcus aureus strain SA75 was isolated from a patient with a purulent skin infection at the First Affiliated Hospital of Wenzhou Medical University (Wenzhou, China). Identification of the isolates was carried out using a VITEK-2 microbiology analyzer according to the manufacturer's instructions (bioMérieux, Marcy l'Etoile, France). $S$. aureus and its derivative strains were grown in tryptic soy broth (TSB, BD) medium with $10 \mathrm{mg} / \mathrm{l}$ chloramphenicol at $37^{\circ} \mathrm{C}$ with shaking at $220 \mathrm{rpm}$. Escherichia coli was grown in Luria broth (LB, Oxoid) medium with appropriate antibiotics (ampicillin at $100 \mathrm{mg} / \mathrm{l}$ and anhydrotetracycline at $50 \mathrm{ng} / \mathrm{ml}$ ).

\section{Construction of $S$. aureus sarX Mutant (SA75 $\Delta$ sarX) and Complemented strain (SA75 $\Delta$ sarX-C)}

The sarX deletion mutant of the SA75 strain was constructed by allelic replacement using the temperature-sensitive plasmid pKOR1. The upstream and downstream fragments of $\operatorname{sar} X$ were amplified from SA75 genomic DNA using the sarX-UP-F/sarX-UP-R and sarX-DOWN-F/sarX-DOWN-R sets of primers (Table 2). The amplified products were digested with $\mathrm{Kpn} \otimes$ and then ligated with T4 DNA ligase to yield the homologous arm fragment with the deletion of sarX gene and then cloned into pKOR1. The recombinant plasmid pKOR1- $\Delta$ sarX was successively transferred into $E$. coli DH5a and DC10B, ultimately electroporated into the $S$. aureus SA75 competent cells. The allelic replacement mutants were selected using a previously described method and were further confirmed by PCR and sequencing [15]. 
To construct the sarX chromosomal complementation strain, the fragments covering the truncated region in the mutant strains were amplified from $S$. aureus strain SA75 genomic DNA using the sarX-F/sarX-R sets of primers (Table 2). The fragments of each gene were digested with restriction enzymes and then cloned into PRB473. The resulting plasmids, PRB473-sarX were electroporated into the mutant strain. The allelic replacement complementation strains were selected using the same method described above and were further confirmed by PCR and sequencing.

\section{RNA isolation and quantitative real-time RT-PCR analysis}

RNA isolation was performed as previously described[16]. Overnight cultures were inoculated to an optical density of 0.01 into fresh TSB medium. Total RNA was isolated and purified using a PureLink RNA Mini Kit (Invitrogen, Carlsbad, CA, United States); then was reverse transcribed into cDNA using a PrimeScript RT reagent kit (TaKaRa, Tokyo, Japan), according to the manufacturer's protocol using the oligonucleotides shown in Table 2. The real-time PCR was carried out using a SsoFas EvaGreen Supermix kit (Bio-Rad, United States) with the Bio-Rad CFX96 Manager software. SA75 wild type strain was used as a control (relative expression $=1$ ), and $g y r B$ was used as a reference gene to investigate genes of interest. RNA transcript levels were calculated by the method of delta delta $\mathrm{Ct}(\Delta \Delta \mathrm{Ct})$ [17]. Data analysis was carried out using Bio-Rad CFX software. Each reaction was performed in triplicate.

\section{Biofilm formation and analysis}

Biofilm formation was determined by the microtiter plate assay based on a previously described method[18]. The overnight cultures were diluted 1:200 in fresh TSB. Two hundred microliter of the diluted cultures of different bacteria were pipetted into sterile 96-well polystyrene plates (BD Biosciences) with three attached wells of each bacteria, then incubated overnight at $37^{\circ} \mathrm{C}$ without shaking. Then, the wells were washed gently three times with phosphate-buffered saline (PBS) (to remove nonadherent cells). Methanol (99.5\%) was used to stabilized biofilms. The wells were stained with $200 \mu \mathrm{l}$ of a $1 \%(\mathrm{w} / \mathrm{v})$ crystal violet (Sigma) for $10 \mathrm{~min}$, and then again washed three times with water. After drying, 30\% glacial acetic acid was used to release the biofilm into the solution. The optical density at $600\left(\mathrm{OD}_{600}\right)$ was recorded.

\section{Scanning Electron Microscopy}

Biofilm formed on glass coverslips ( $10 \mathrm{~mm}$ in diameter) was observed by scanning electron microscopy (SEM) as previously described[19]. Briefly, overnight cultures were diluted 1:200 with TSB. A glass disk was put in advance into the bottom of the sterile flat bottomed polystyrene plates (Costar 3524; Corning, $\mathrm{NY}$, United States) and $1 \mathrm{ml}$ of the cell suspensions was added into the wells, with static culture at $37^{\circ} \mathrm{C}$ for $24 \mathrm{~h}$. The bacteria were cultured under the same conditions for colony counting to ensure that the amount of bacteria was consistent and the next experiment was carried out. Each well was rinsed three times with sterile PBS, each time for 10 min with slight shaking. Biofilms formed on the glasses were fixed with $2.5 \%$ glutaraldehyde in $0.1 \mathrm{M}$ sodium cacodylate buffer $(\mathrm{pH} 7.4)$ by incubation for $2 \mathrm{~h}$ at room temperature. Next, the coverslips were washed three times with sterile PBS for 15 min. Thereafter, $1 \%$ 
osmium tetroxide was used for a post-fixation step for $2 \mathrm{~h}$ at $4^{\circ} \mathrm{C}$, followed by three times washed with distilled water for $10 \mathrm{~min}$ each, and then dehydrated in a series of ascending ethanol baths $(25,50,75,95$ and $100 \%$ ) for $10 \mathrm{~min}$ each. The coverslips were placed in a freeze-dried apparatus for $5 \mathrm{~h}$, and the gold spray was taken out for $3 \mathrm{~min}$. The observations were usually performed with a scanning electron microscope (FEI Quanta 200; United States). The experiment was repeated three times.

\section{Triton X-100 Induced Autolysis}

Autolysis assays were performed as described by Brunskill and Bayles[20]. The overnight cultures were cultured in $50 \mathrm{ml}$ TSB containing $1 \mathrm{M} \mathrm{NaCl}$ until they reached the early exponential phase. Following centrifugation $(4000 \mathrm{~g}, 20 \mathrm{~min})$, the cells were washed twice with $50 \mathrm{ml}$ of ice-cold water and resuspended into $50 \mathrm{ml}$ of Tris $-\mathrm{HCl}\left(\mathrm{pH} \mathrm{7.2)}\right.$ containing $0.05 \%$ (vol/vol) TritonX-100. The $\mathrm{OD}_{600}$ value was adjusted to 1.0 , followed by shaking of the culture at $37^{\circ} \mathrm{C}$ for $3 \mathrm{~h}$ and was measured at $30 \mathrm{~min}$ intervals. The experiment was repeated three times.

\section{Western Blot analysis}

The protein expression level of Staphylococcus protein A (SPA) was determined by western blot analysis as previously described[21]. Culture supernatant was collected and washed twice with sterile PBS. Then, supernatant was suspended in $100 \mu \mathrm{l}$ of PBS incubation at $37^{\circ} \mathrm{C}$ for $2 \mathrm{~h}$ with $3 \mu$ of lysostaphin ( 1 $\mathrm{mg} / \mathrm{ml})$ and centrifuged $\left(8000 \mathrm{~g}, 30 \mathrm{~min}, 4^{\circ} \mathrm{C}\right)$. The protein concentration of each sample was determined by Bradford dye binding method. The samples were separated by $12 \%$ sodium dodecyl sulphate polyacrylamide gel (SDS-PAGE) and electrotransferred onto a polyvinylidene difluoride (PVDF) membrane. After blocking with $5 \%$ nonfat milk in PBST buffer at room temperature for $1 \mathrm{~h}$, the membrane was incubated with Anti-Protein A (HRP) antibody at a 1/5000 dilution. The image was visualized using the ECL Western blotting assay kit (GE Healthcare) and the Chemi doc TM XRS system (Bio rad, USA).

\section{PIA/PNAG detection}

Polysaccharide intercellular adhesion (PIA) extracted from S. aureus was detected by a dot blot assay with a germ agglutinin horseradish peroxidase (WGA-HRP) conjugate according to a previously described protocol[22]. Briefly, overnight cultures were diluted to $10^{7}$ colony-forming units (CFU) and added to $2 \mathrm{ml}$ TSB at a ratio of 1:100 in the six-well polystyrene plates. An equal number of cells from each culture was resuspended in $0.5 \mathrm{M} \mathrm{EDTA}(\mathrm{pH} 8.0)$ and incubated for $5 \mathrm{~min}$ at $100^{\circ} \mathrm{C}$ and centrifuged, and $40 \mu \mathrm{l}$ of the supernatant was incubated with $10 \mu \mathrm{l}$ of proteinase $\mathrm{K}\left(20 \mathrm{mg} / \mathrm{ml}\right.$ ) (Sangon Biotech) at $37^{\circ} \mathrm{C}$ for $2 \mathrm{~h}$ to minimize nonspecific background. Pipette $10 \mu \mathrm{l}$ of the extracted PIA sample onto the PVDF membrane after formaldehyde treatment, keep the PVDF membrane moist during the spotting process, and dry the membrane at room temperature after spotting. The membrane was blocked with $3.5 \%$ bovine serum albumin (BSA) in PBS with $0.1 \%$ Tween 20. The membrane was placed in a dish containing wheat germ agglutinin conjugated to WGA-HRP, and incubated at $37^{\circ} \mathrm{C}$ for $1 \mathrm{~h}$. Then color developed with Pierce enhanced chemiluminescence (ECL) Western blotting substrate (Thermo Scientific, Rockford, IL). 


\section{Statistical analyses}

All experiments were performed in biological triplicate. Statistical analysis was performed using SPSS statistical software (version23; IBM SPSS Statistic) and GraphPad Prism 8 (version 8.00, La Jolla, CA, United States). For the comparison of two groups, unpaired $t$ test was used; for three or more groups, 1way or 2-way analysis of variance was used, as appropriate. A value of $\mathrm{P}<0.05$ was considered statistically significant.

\section{Results}

\section{The sarX mutation reduces $S$. aureus biofilm formation}

We evaluated the effect of SarX on the biofilm formation capacity of $S$. aureus by comparing the $\Delta$ sarX mutant with SA75 $\triangle$ sarX-C and SA75 strains. The decreased biofilm formation of the sarX mutant strain could not be the consequence of the growth defect. There was no significant difference of the growth rates among SA75, SA75- $\Delta$ sarX, and SA75- $\triangle$ sarX-C strains under the same inoculation and growth conditions (Fig.1C). In comparison with SA75, SA75- $\triangle$ sarX displayed a marked reduction in biofilm formation (Fig. 1a). In contrast, SA75- $\triangle$ sarX-C formed biofilms similar to the SA75 strain when the strains were incubated under the same conditions. The semi quantification result for biofilm was shown in Fig. $1 \mathrm{~b}$. The mature biofilm of the SA75- $\Delta$ sarX strain was significantly increased compared to that of SA75 strain (3.31-fold, $P<0.05)$. However, the density of the mature biofilm of SA75- $\Delta$ sar $X-C$ was similar to the wild type strain.

\section{Scanning Electron Microscopy}

Scanning electron microscopy can be used to observe the formation of biofilm. As shown in Fig. 3, SA75 and SA75- $\Delta$ sarX-C could be seen to accumulate and formed biofilm obviously, the bacteria clustered together, and the bacteria and bacteria adhere to each other to form a compact biofilm structure. However, the thickness of mature biofilms decreased in SA75- $\Delta$ sarX strain. The biofilm formed by strain of SA75- $\triangle$ sarX was significantly thinner than SA75. Similar to the biofilm formation results, the biofilm formation of SA75- $\triangle$ sarX-C was similar to that of SA75, but there was no adhesion between the colonies, and the profile of a single colony can be clearly observed on the surface of the agglomerated biofilm.

\section{The reduced biofilm formation of the sarX mutant strain is PIA dependent}

The PIA production was determined semi quantitatively with WGA-HRP conjugate sing a dot blot 96 system (Figure 2a). Consistent with the result of RT-PCR, the sarX mutant strain displayed much weaker PIA production compared with the wild type strain, while the complemented strain exhibited restored PIA production. In order to investigate the effect of gene sarX on biofilm matrix production, the transcription icaADBC operon in the wild type SA75, SA75- $\triangle$ sarX and SA75- $\triangle$ sarX-C strains using real-time RT-PCR, and the release of PIA was determined. As shown in Fig. $2 \mathrm{~b}$, the expression of $i c a A$ in SA75- $\Delta$ sarX was 
significantly reduced, indicating that knockout of $\operatorname{sar} X$ would inhibit the expression of $i c a A$. The expression level of SA75- $\Delta$ sarX-C can be restored to the wild level.

\section{The autolysis capacity}

Autolysis of cells resulted in the release of intracellular eDNA, which can promote adhesion and aggregation between cells and promote the formation of biofilms. We want to know whether the decreased biofilm formation of sarX strain was associated with autolysis or not. The autolytic capacity of SA75, SA75- $\Delta$ sarX and SA75- $\Delta$ sarX-C were assessed by TritonX-100 introduction. Staphylococcus epidermidis $1457 \triangle$ at/E was used as the control strain. As shown in Fig. 2c, no difference was found between SA75- $\Delta$ sarX and its parent strains in the TritonX-100 induced autolysis.

\section{Detection of Staphylococcus protein A (SPA) in S. aureus}

Staphylococcus protein A (SPA) is an important adhesion protein of $S$. aureus. It can enhance the adhesion between bacteria and promote the formation of biofilms. As shown in figure $4 \mathrm{a}$, we extracted the SPA of three strains of bacteria (SA75, SA75- $\Delta$ sarX and SA75- $\Delta$ sarX-C), and carried out while keeping the total amount of protein loaded SDS-PAGE, Western Blot method was used to detect the protein expression of SPA. The production level of SPA in sarX mutant strain was much lower than the SA75 strain and sarX complemented strains. The results showed (Figure 4b) that the expression of spa in SA75- $\Delta$ sar $X$ was significantly reduced, indicating that knockout of $\operatorname{sar} X$ would inhibit the expression of spa. The expression level of SA75- $\triangle$ sar $X-C$ can be restored to the wild level.

\section{Discussion}

The production of PIA is an important contributing factor to biofilm formation by staphylococci. icaADBC encoding PIA are subject to regulation by numerous factors. Transcription of $i c a A D B C$ is also subject to positive regulation by SarA. SarA increased biofilm development by suppressing the transcription of either a protein involved in the turnover of PIA/PNAG or a repressor of its synthesis. The Sar family of proteins is composed of at least 11 different proteins. Manna and Cheung found that sarX affected biofilm formation in $S$. aureus 8325-4, while did not in $S$. aureus RN6390 [10]. It is still unknown that reasons of the difference exists between RN6390 and 8325-4. Because they are closely related strains derived from the same parent strain (NCTC8325). In present study, we found that deletion of sarX failed to stimulate biofilm formation, while complement sarX mutant isolate exhibited the same levels of biofilm formation as the clinical isolate $S$. aureus SA75. In brief, our results revealed a positive role for sarX in $S$. aureus biofilm regulation. In addition, RT-PCR results revealed that deletion of sarX was associated with a 2 -fold reduction in icaA transcription, which was complemented by sarX. PIA production was increased in SA75- $\Delta$ sarX strain. The results revealed here that sarX mutant in $S$. aureus biofilm formation was related to the production of PIA and not to that of eDNA. Similarly, David Cue et al. demonstrate that Rbf and SarX represent a regulatory cascade that promotes PIA-dependent biofilm formation in S. aureus [23]. Rbf, member of the AraC/XylS family of transcriptional regulators, increase icaADBC expression by upregulating transcription of sarX. SarX, in turn, activates icaADBC expression[23]. 
The spa gene encodes protein A, a multifunctional cell wall protein that binds immunoglobulins, thereby inhibiting opsonophagocytosis. In present study, RT-PCR results showed that deletion of sarX was associated with a 1.8-fold reduction in spa transcription, which was complemented by sarX. Expression of Spa protein was decreased in sarX mutant strain. Previous study found that function for Spa in the proportion of cell to cell interactions and biofilm formation by investigating the protein dependent biofilm production[12]. Therefore, we speculated biofilm formation in S. aureus may associated with spa. However, many questions remain as we do not completely understand what signals induce spa expression, how Spa regulates sarX expression, or precisely how SarX promotes spa transcription.

In conclusion, sarX promoted biofilm production may mediated primarily through increased ica operon expression and PIA production. Deletion of $s a r X$ was associated with reduction in spa transcription.

\section{Declarations}

\section{Ethics approval and consent to participate}

This study was approved by the Ethics Committee of the first Affiliated Hospital of Wenzhou Medical University. All methods were carried out in accordance with relevant guidelines and regulations. All experimental protocols were approved by a named institutional and/or licensing committee. Informed consent was obtained from all subjects.

\section{Consent for publication}

Not applicable

\section{Acknowledgements}

The authors are grateful to the First Affiliated Hospital of Wenzhou Medical University.

\section{Authors' contributions}

$Z H, Y G, J Y, L R, Q Z$ and $Y X$ performed the laboratory measurements. BW and $X W$ made substantial contributions to conception and design. ZH drafted the manuscript. YG and FY revised the manuscript critically for important intellectual content. All authors read and approved the final manuscript.

\section{Author's information}

Member of Shanghai Pulmonary Hospital, Tongji University School of Medicine: Yinjuan Guo, Bingjie Wang, Xiaocui Wu, and Fangyou Yu.

Member of the First Affiliated Hospital of Wenzhou Medical University: Zhihao Hao, Jingyi Yu, and Lulin Rao.

Member of the Nanchang University: Qing Zhan and Yanlei Xu 


\section{Funding}

This study was supported by grants from Natural Science fund of China

(81871704). It supported the each section of this study, including design of

the study and collection, analysis, and interpretation of data and in writing

the manuscript.

\section{Availability of data and materials}

The datasets used during the current study are available from the corresponding author upon reasonable request. All the data is included in this published article.

\section{Consent for publication}

Not applicable.

\section{Competing interests}

The authors declare they have no competing interests.

\section{Abbreviations}

PIA: polysaccharide intercellular adhere

MarR: multiple antibiotic resistance regulator

Bap: biofilm-associated protein

SA75: Staphylococcus aureus strain SA75

TSB: tryptic soy broth

LB: Luria broth

SEM: scanning electron microscopy

SPA: Staphylococcus protein A

OD: optical density

SDS-PAGE: sodium dodecyl sulphate polyacrylamide gel

PVDF: polyvinylidene difluoride 
CFU: colony-forming units

BSA: ovine serum albumin

\section{References}

1. Zhao G, Jiang K, Wu H, Qiu C, Deng G, Peng X: Polydatin reduces Staphylococcus aureus lipoteichoic acid-induced injury by attenuating reactive oxygen species generation and TLR2-NFkappaB signalling. J Cell Mol Med 2017, 21(11):2796-2808.

2. Wertheim HF, Melles DC, Vos MC, van Leeuwen W, van Belkum A, Verbrugh HA, Nouwen JL: The role of nasal carriage in Staphylococcus aureus infections. The Lancet Infectious diseases 2005, 5(12):751-762.

3. Furukawa S, Kuchma SL, O'Toole GA: Keeping their options open: acute versus persistent infections. Journal of bacteriology 2006, 188(4):1211-1217.

4. Davies D: Understanding biofilm resistance to antibacterial agents. Nature reviews Drug discovery 2003, 2(2):114-122.

5. McCarthy AJ, Lindsay JA: Genetic variation in Staphylococcus aureus surface and immune evasion genes is lineage associated: implications for vaccine design and host-pathogen interactions. $B M C$ microbiology 2010, 10:173.

6. Boles BR, Horswill AR: Staphylococcal biofilm disassembly. Trends Microbio/2011, 19(9):449-455.

7. Skurnik D, Cywes-Bentley C, Pier GB: The exceptionally broad-based potential of active and passive vaccination targeting the conserved microbial surface polysaccharide PNAG. Expert Rev Vaccines 2016, 15(8):1041-1053.

8. Jefferson KK, Pier DB, Goldmann DA, Pier GB: The teicoplanin-associated locus regulator (TcaR) and the intercellular adhesin locus regulator (IcaR) are transcriptional inhibitors of the ica locus in Staphylococcus aureus. J Bacterio/ 2004, 186(8):2449-2456.

9. Brandenberger M, Tschierske M, Giachino P, Wada A, Berger-Bachi B: Inactivation of a novel threecistronic operon tcaR-tcaA-tcaB increases teicoplanin resistance in Staphylococcus aureus. Biochim Biophys Acta 2000, 1523(2-3):135-139.

10. Manna AC, Cheung AL: Expression of SarX, a negative regulator of agr and exoprotein synthesis, is activated by MgrA in Staphylococcus aureus. J Bacteriol 2006, 188(12):4288-4299.

11. Rowe SE, Mahon V, Smith SG, O'Gara JP: A novel role for SarX in Staphylococcus epidermidis biofilm regulation. Microbiology (Reading) 2011, 157(Pt 4):1042-1049.

12. Merino N, Toledo-Arana A, Vergara-Irigaray M, Valle J, Solano C, Calvo E, Lopez JA, Foster TJ, Penades JR, Lasa I: Protein A-mediated multicellular behavior in Staphylococcus aureus. J Bacteriol 2009, 191(3):832-843.

13. Foster TJ, Geoghegan JA, Ganesh VK, Hook M: Adhesion, invasion and evasion: the many functions of the surface proteins of Staphylococcus aureus. Nat Rev Microbio/ 2014, 12(1):49-62. 
14. Schneewind O, Model P, Fischetti VA: Sorting of protein A to the staphylococcal cell wall. Cell 1992, 70(2):267-281.

15. Bae T, Schneewind O: Allelic replacement in Staphylococcus aureus with inducible counter-selection. Plasmid 2006, 55(1):58-63.

16. Wolz C, Goerke C, Landmann R, Zimmerli W, Fluckiger U: Transcription of clumping factor A in attached and unattached Staphylococcus aureus in vitro and during device-related infection. Infect Immun 2002, 70(6):2758-2762.

17. Montgomery CP, Boyle-Vavra S, Roux A, Ebine K, Sonenshein AL, Daum RS: CodY deletion enhances in vivo virulence of community-associated methicillin-resistant Staphylococcus aureus clone USA300. Infect Immun 2012, 80(7):2382-2389.

18. C H, C G, F P-R, F G: Characterization of Tn917 insertion mutants of Staphylococcus epidermidis affected in biofilm formation. Infection and immunity 1996, 64(1):277-282.

19. V P, C M, R M, S V, WE P, JK K, J VE: The role of sigmaB in persistence of Staphylococcus epidermidis foreign body infection. Microbiology (Reading, England) 2008, 154(null):2827-2836.

20. Brunskill EW, Bayles KW: Identification and molecular characterization of a putative regulatory locus that affects autolysis in Staphylococcus aureus. J Bacterio/ 1996, 178(3):611-618.

21. Jelsbak L, Ingmer H, Valihrach L, Cohn MT, Christiansen MH, Kallipolitis BH, Frees D: The chaperone ClpX stimulates expression of Staphylococcus aureus protein A by Rot dependent and independent pathways. PLoS One 2010, 5(9):e12752.

22. SE C, C G, NF S, WW N, F G: The intercellular adhesion (ica) locus is present in Staphylococcus aureus and is required for biofilm formation. Infection and immunity 1999, 67(10):5427-5433.

23. Cue $D$, Lei MG, Lee CY: Activation of sarX by Rbf is required for biofilm formation and icaADBC expression in Staphylococcus aureus. J Bacteriol 2013, 195(7):1515-1524.

\section{Tables}

Table 1. Bacterial strains and plasmids used in this study 


\begin{tabular}{|c|c|c|}
\hline $\begin{array}{l}\text { Strains and } \\
\text { Plasmids }\end{array}$ & Description ${ }^{a}$ & Source \\
\hline \multicolumn{3}{|l|}{ Strains } \\
\hline $\begin{array}{l}\text { S. aureus } \\
\text { SA75 }\end{array}$ & Wild type, clinical MRSA strain & $\begin{array}{l}\text { The First Affiliated Hospital of } \\
\text { Wenzhou Medical University }\end{array}$ \\
\hline $\mathrm{SA75} \triangle S A R X$ & Isogenic SARX deletion mutant in SA75 & This study \\
\hline $\begin{array}{l}\text { SA75 } \triangle S A R X- \\
\text { C }\end{array}$ & SARX mutant complemented with $\mathrm{pRBSARX}$ & This study \\
\hline DH5 & Escherichia coli isolates, clone host strain & Laboratory stock \\
\hline DC10B & $\begin{array}{l}\text { Escherichia coli isolates, dam }+\Delta \mathrm{dcm} \text { hsdRMS } \\
\text { endA1 recA1; clone host strain }\end{array}$ & Laboratory stock \\
\hline \multicolumn{3}{|l|}{ Plasmids } \\
\hline pKOR1 & $\begin{array}{l}\text { Shuttle cloning vector, temp sensitive }\left(\mathrm{Cm}^{\mathrm{R}} \text {, }\right. \\
\left.A m p^{R}\right)\end{array}$ & Laboratory stock \\
\hline pRB473 & Shuttle cloning vector $\left(\mathrm{Cm}^{\mathrm{R}}\right)$ & Laboratory stock \\
\hline
\end{tabular}

${ }^{a} \mathrm{Cm}^{\mathrm{R}}$, chloramphenicol resistance; $\mathrm{Amp}^{\mathrm{R}}$, ampicillin resistance.

Table 2. Primers used in this study 


\begin{tabular}{|c|c|c|}
\hline Primer & Primer sequence $\left(5^{\prime}-3^{\prime}\right)$ & Note \\
\hline sarX-F & AAGATAATAATGACAGAAGC & \\
\hline sarX-R & AAAATTGTTCTACATCTTCA & \\
\hline sarX-UP-F & GGGGACAAGTTTGTACAAAAAAGCAGGCTAGCGACTTAAATTCGATTCGTTA & attB1 \\
\hline sarX-UP-R & CGGGGTACCGTCTTTCTCATTTGTTTTTAATACG & Kpnl \\
\hline $\begin{array}{l}\text { sarX-DOWN- } \\
\mathrm{F}\end{array}$ & CGGGGTACCTTGAAATTGAGTGTCGAAAGCATAG & Kpnl \\
\hline $\begin{array}{l}\text { sarX-DOWN- } \\
\mathrm{R}\end{array}$ & GGGGACCACTTTGTACAAGAAAGCTGG GTTCCATTGTTCTGCTGATT & attB2 \\
\hline sarX-RT-F & AACATTGCTTGGCTTCTAT & \\
\hline sarX-RT-R & AATCTAGCTCATCCATTGC & \\
\hline sarX-C-F & CGGAATTCCG ACACCTTGATATGTATTGCA & EcoRI \\
\hline $\operatorname{sar} X-\mathrm{C}-\mathrm{R}$ & CGGGATCCCG CATTATTGAACTACGATTCC & $\mathrm{BamHI}$ \\
\hline icaA-RT-F & CTTGGATGCAGATACTATCG & \\
\hline icaA-RT-R & GCGTTGCTTCCAAAGACCTC & \\
\hline spa-RT-F & CAATTCTAGCTATTATCACTT & \\
\hline spa-RT-R & ATTAATACCCCCTGTATGTA & \\
\hline
\end{tabular}

\section{Figures}


$\mathbf{a}$

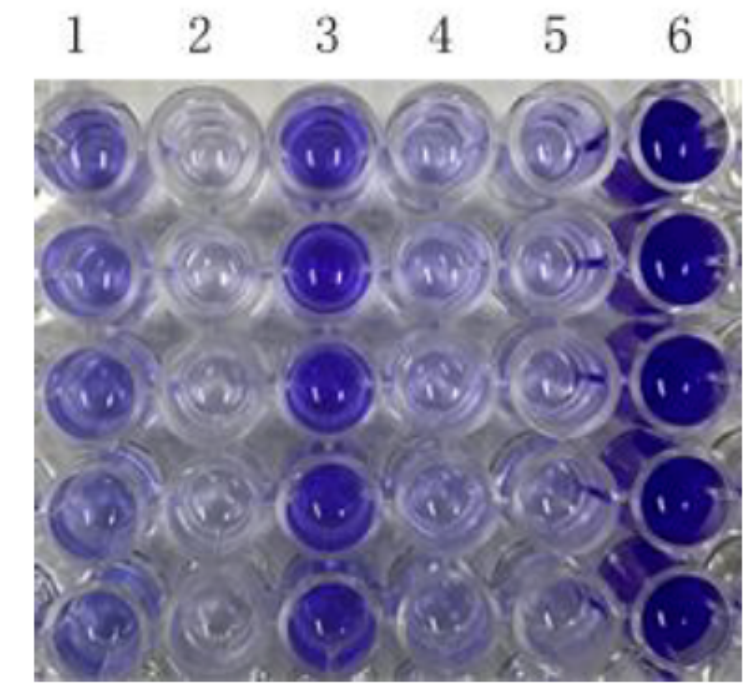

b

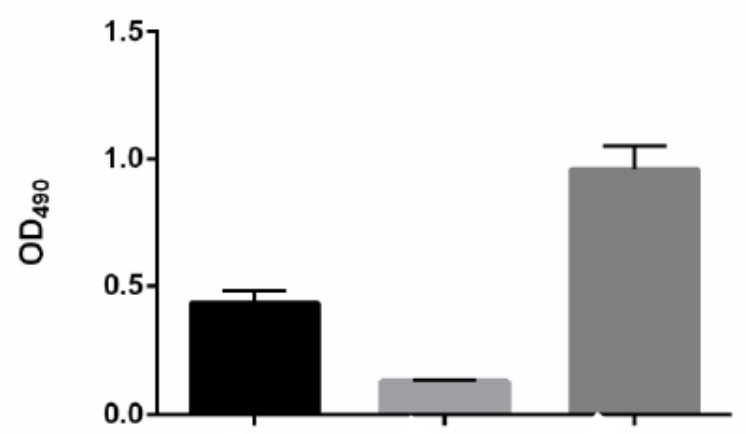

c

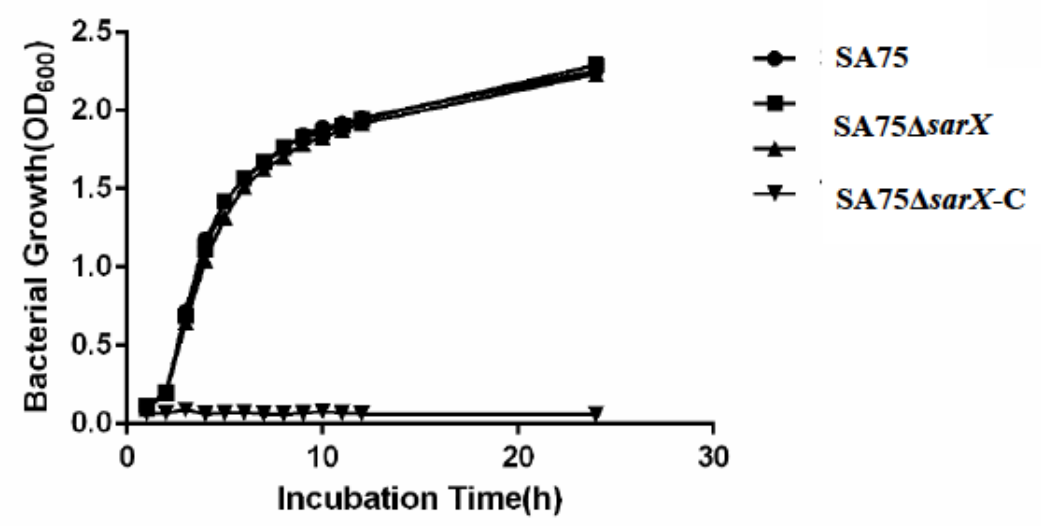

\section{Figure 1}

The sarX mutation reduces S. aureus biofilm formation. (a): Biofilm formation by SA75 wild-type (1), SA75 $\Delta$ sarX (2) and SA75 $\Delta$ sarX-C (3) strains on microtiter plates. 4: blank control. 5: negative control. 6: positive control. (b): Biofilm quantification. Glacial acetic acid (33\%) was used to release the biofilms into solution. Each experiment was repeated three times, and the data represent means + standard deviations. (c): Growth of the SA75 wild-type, $\Delta$ sarX mutant and chromosomal complemented strains. 
a

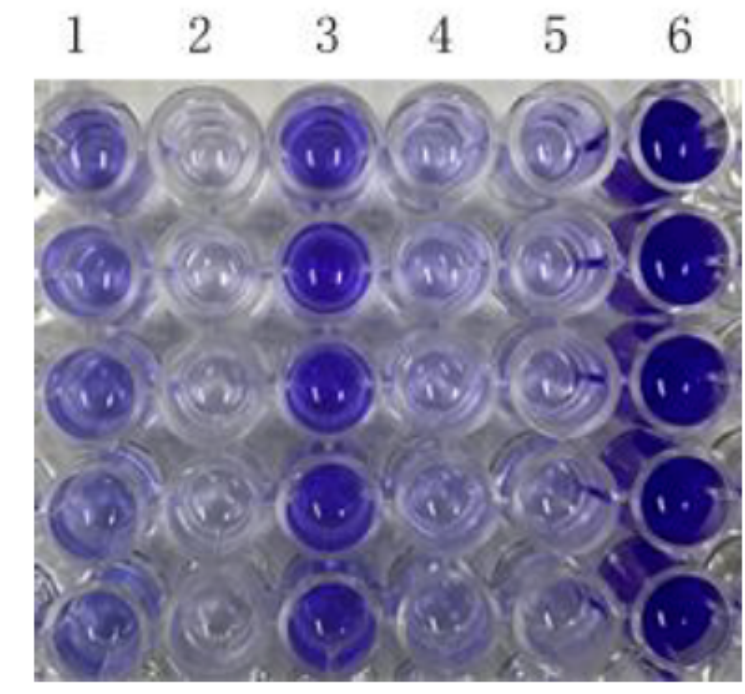

b

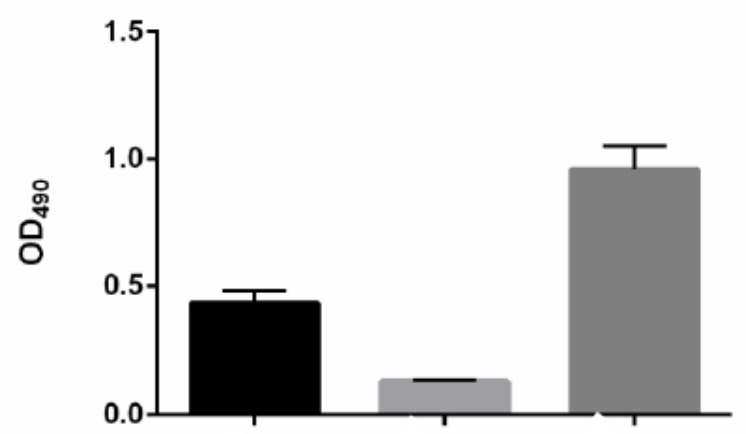

c

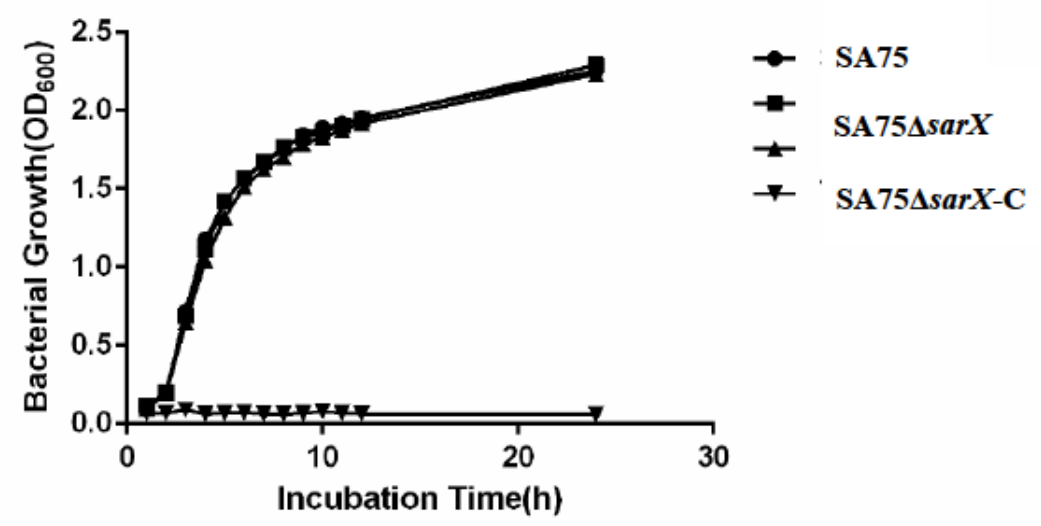

\section{Figure 1}

The sarX mutation reduces S. aureus biofilm formation. (a): Biofilm formation by SA75 wild-type (1), SA75 $\Delta$ sarX (2) and SA75 $\Delta$ sarX-C (3) strains on microtiter plates. 4: blank control. 5: negative control. 6: positive control. (b): Biofilm quantification. Glacial acetic acid (33\%) was used to release the biofilms into solution. Each experiment was repeated three times, and the data represent means + standard deviations. (c): Growth of the SA75 wild-type, $\Delta$ sarX mutant and chromosomal complemented strains. 
$\mathbf{a}$
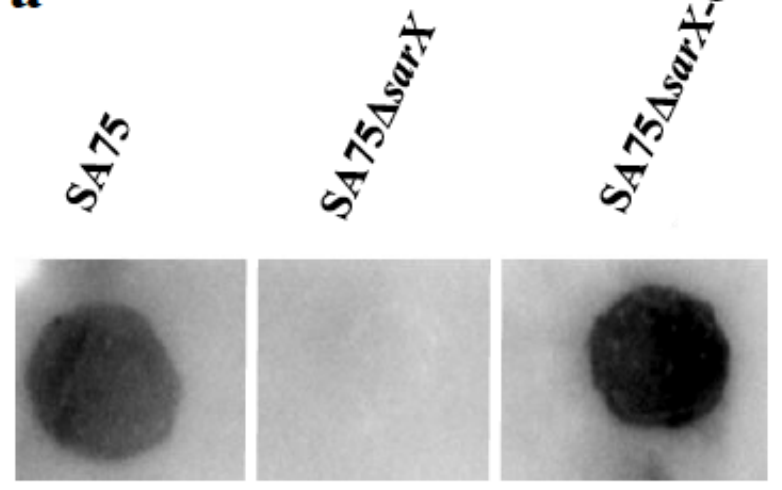

c b

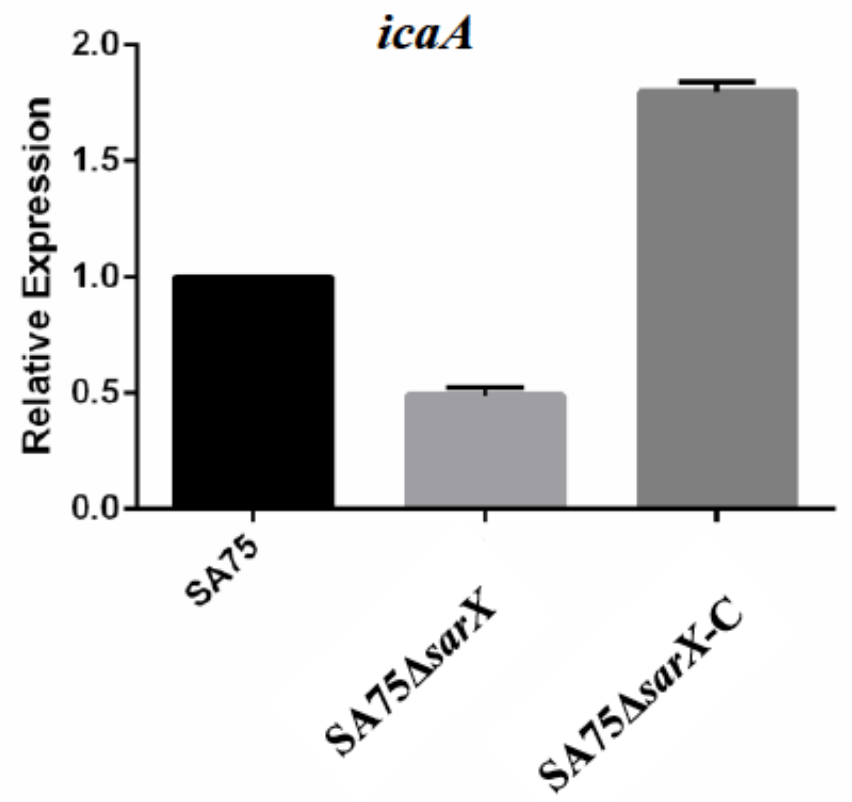

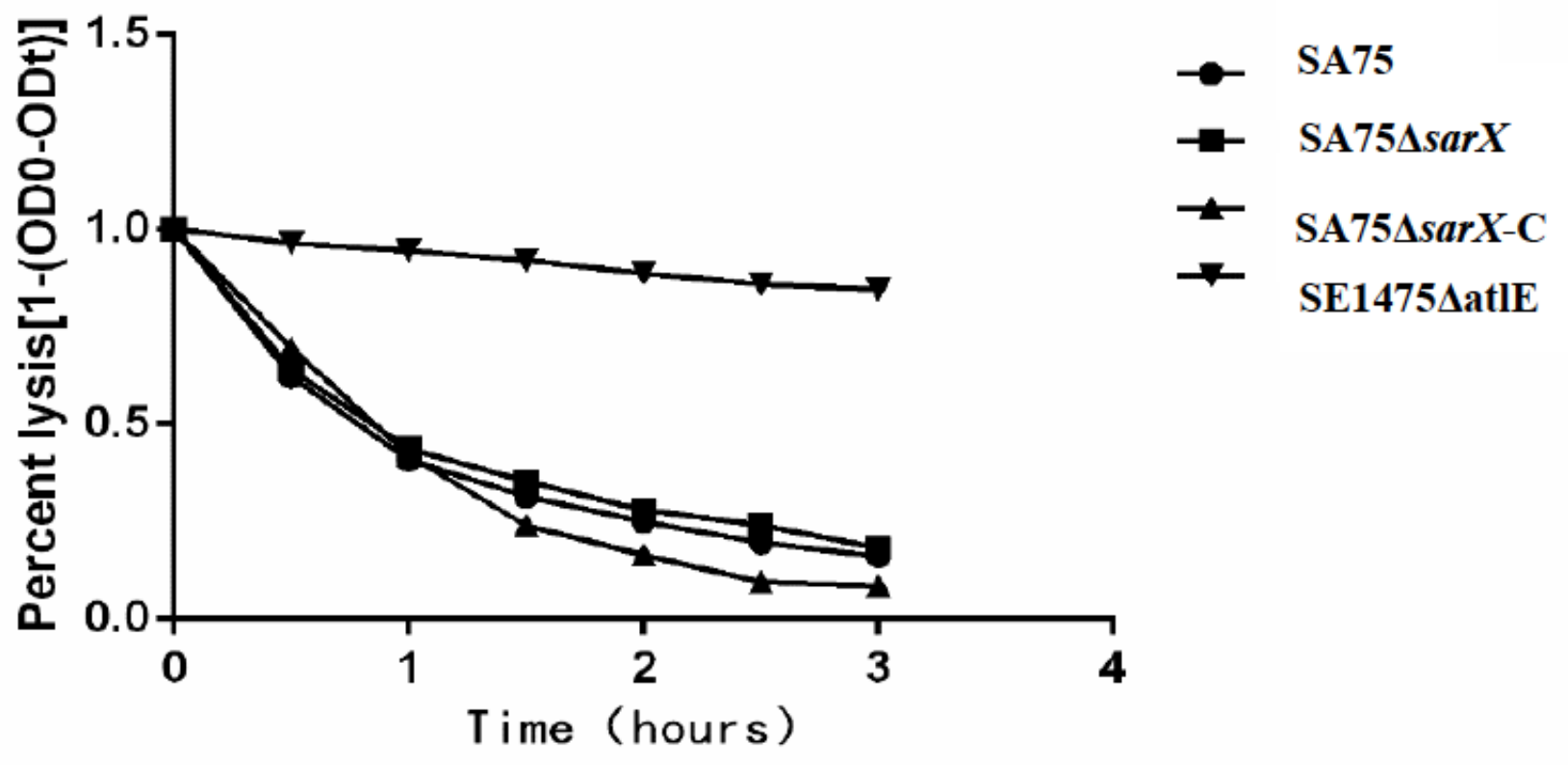

Figure 2

The reduced biofilm formation of the sarX mutant is PIA dependent. (a) PIA biosynthesis was semi quantified using a dot blot assay with WGA. (b) Reverse transcription-PCR (RT-PCR) quantification of the effect of sarX mutant on icaA gene expression by the SA75 strain. Comparison of the autolytic abilities of SA75 wild-type, SA75 $\Delta$ sarX and SA75 $\Delta$ sarX-C strains. TritonX-100 induced 
$\mathbf{a}$
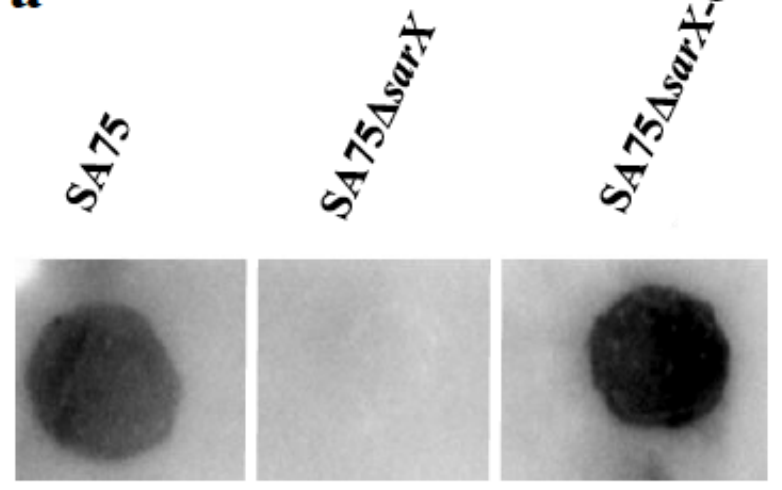

c b

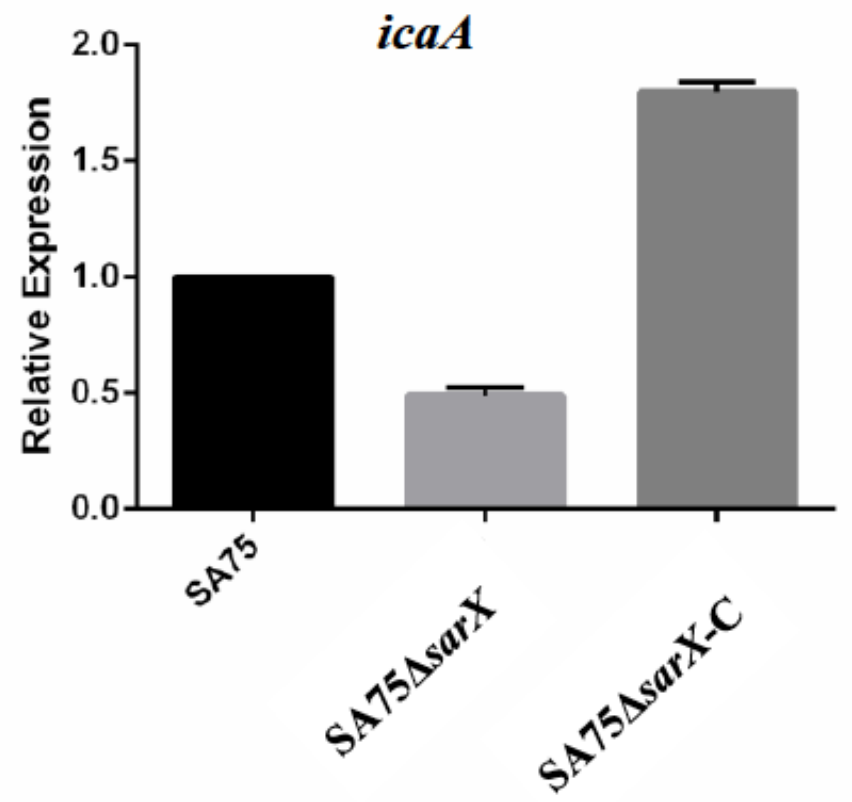

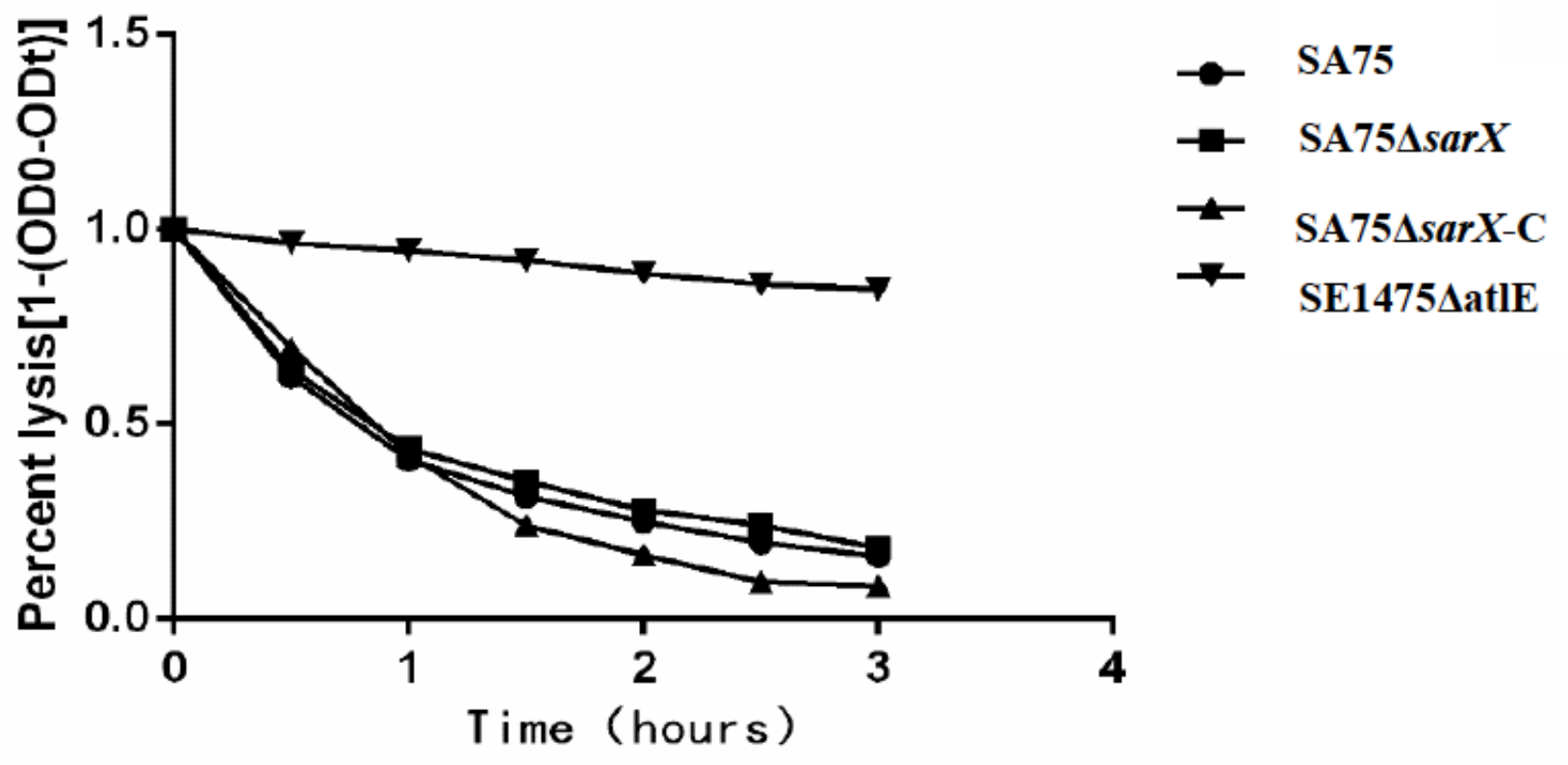

Figure 2

The reduced biofilm formation of the sarX mutant is PIA dependent. (a) PIA biosynthesis was semi quantified using a dot blot assay with WGA. (b) Reverse transcription-PCR (RT-PCR) quantification of the effect of sarX mutant on icaA gene expression by the SA75 strain. Comparison of the autolytic abilities of SA75 wild-type, SA75 $\Delta$ sarX and SA75 $\Delta$ sarX-C strains. TritonX-100 induced 
2000X

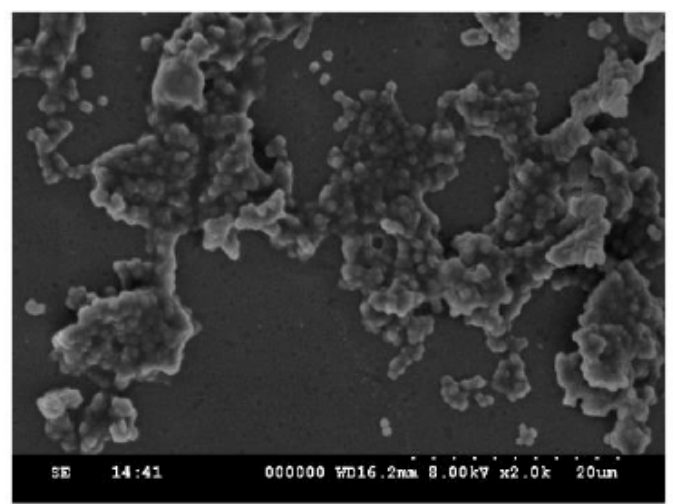

SA75

\section{SA75 $\Delta s a r X$}

SA75 $\Delta$ sarX-C
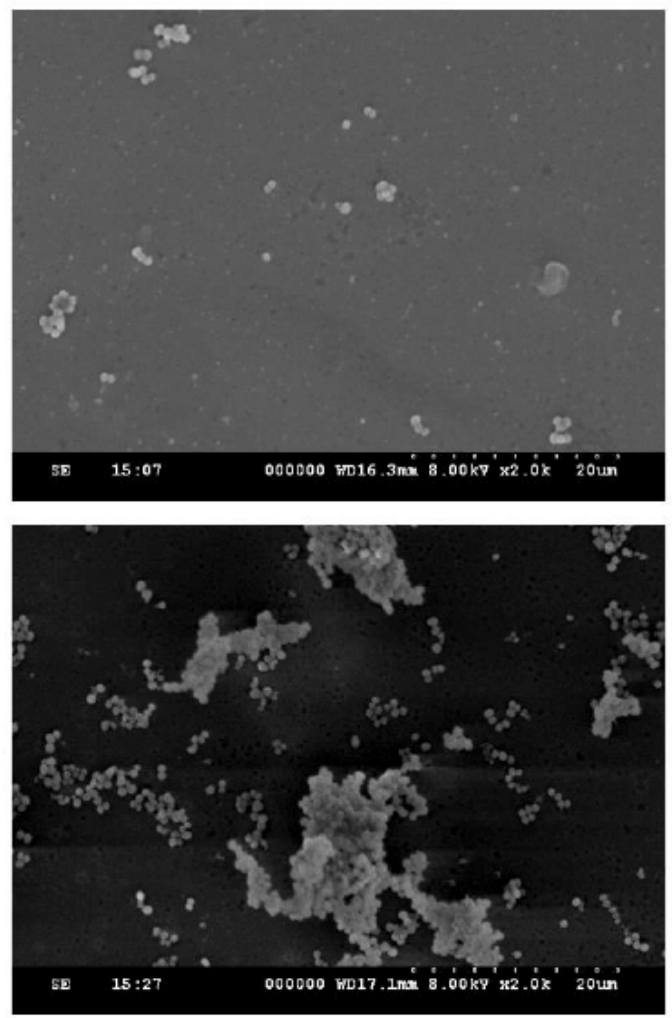

$5000 \mathrm{X}$
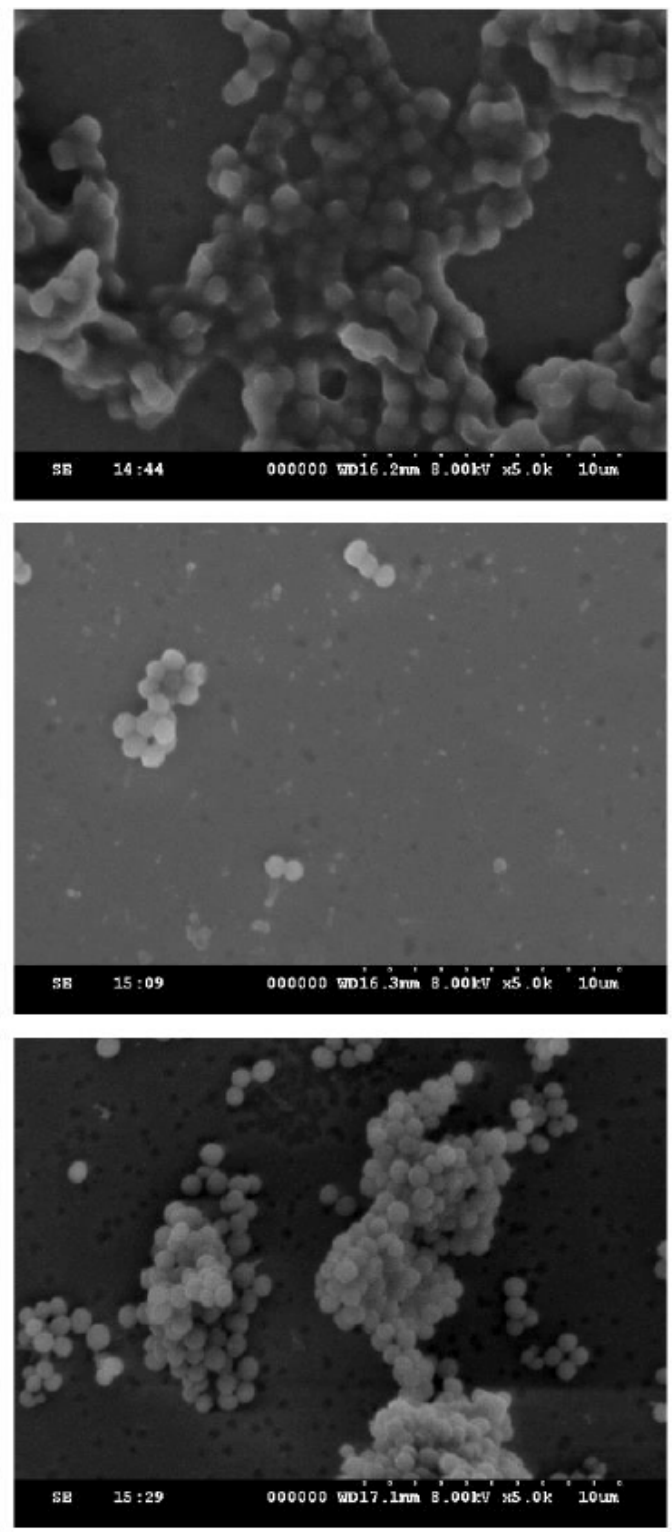

\section{Figure 3}

Scanning electron microscope study. Transmission electron micrograph of biofilm in mature stage of wild type (SA75), isogenic sarX mutant, and SA75 $\triangle$ sarX-C strains. The images on the left side were magnified 2,000 times, and images on the right sides were magnified 5,000 times. 
2000X

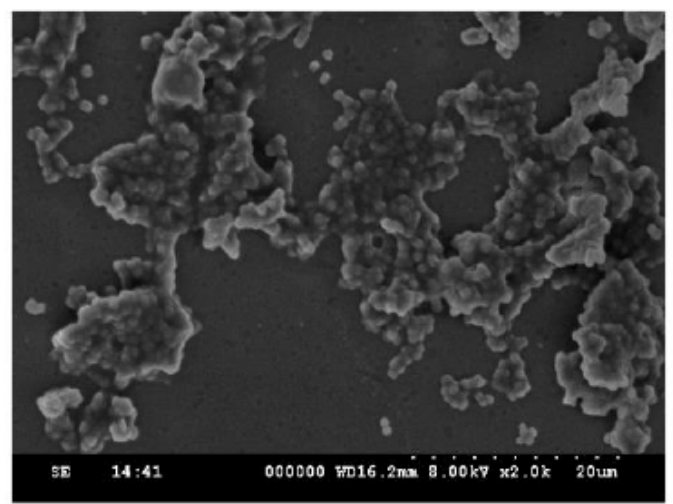

SA75

\section{SA75 $\Delta s a r X$}

SA75 $\Delta$ sarX-C
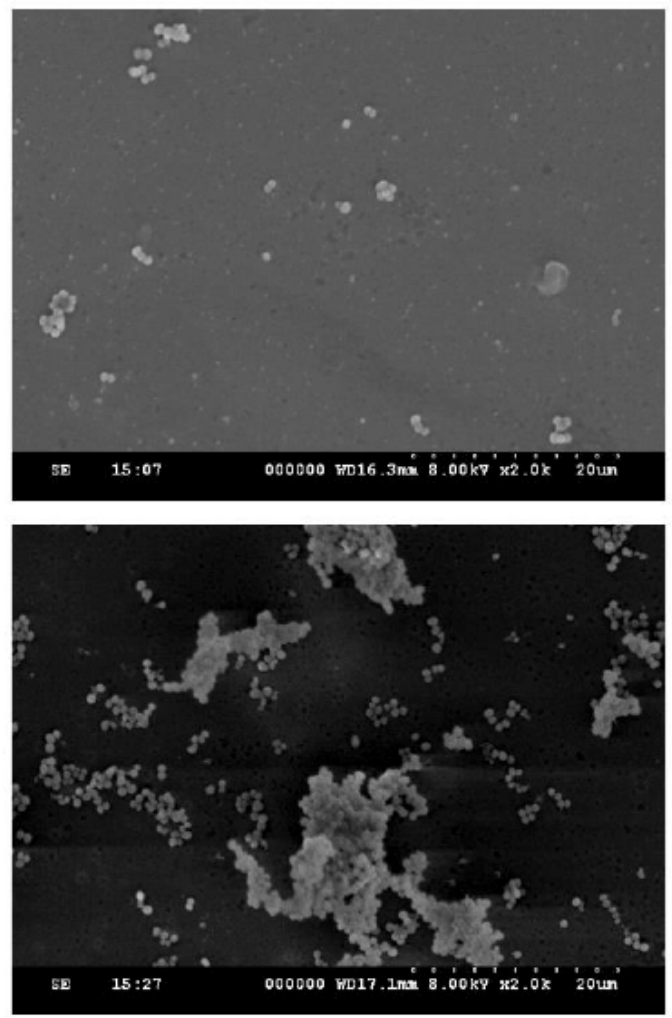

$5000 \mathrm{X}$
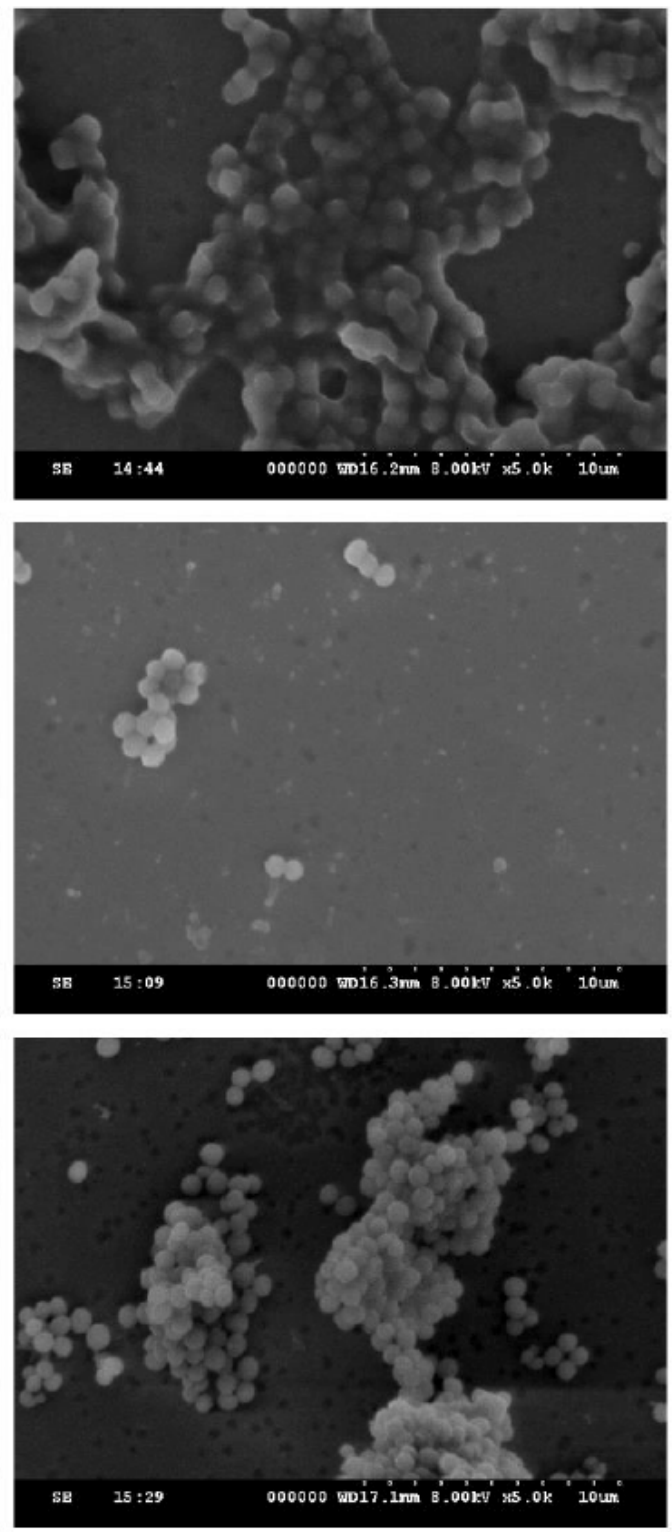

\section{Figure 3}

Scanning electron microscope study. Transmission electron micrograph of biofilm in mature stage of wild type (SA75), isogenic sarX mutant, and SA75 $\triangle$ sarX-C strains. The images on the left side were magnified 2,000 times, and images on the right sides were magnified 5,000 times. 
a
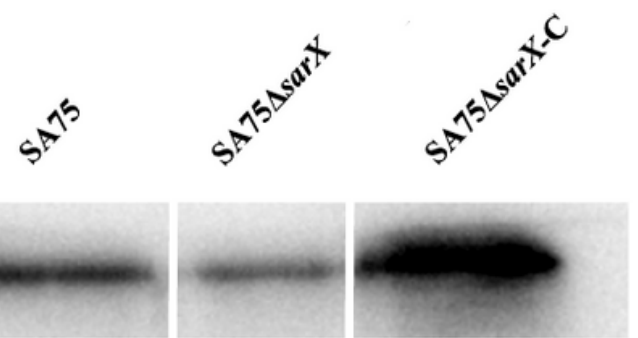

b

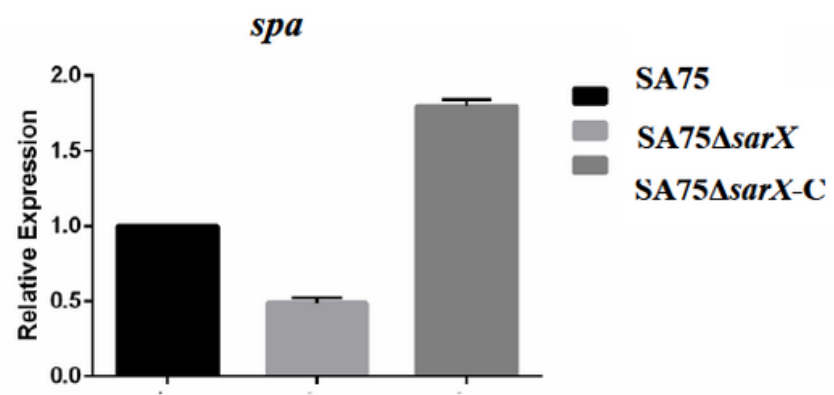

Figure 4

SPA expression levels of SA75, SA75- $\Delta$ sarX, and SA75- $\Delta$ sarX-C RT-PCR results of spa

$\mathbf{a}$
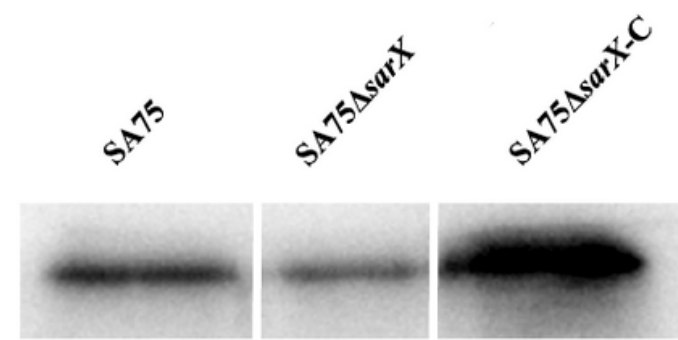

b

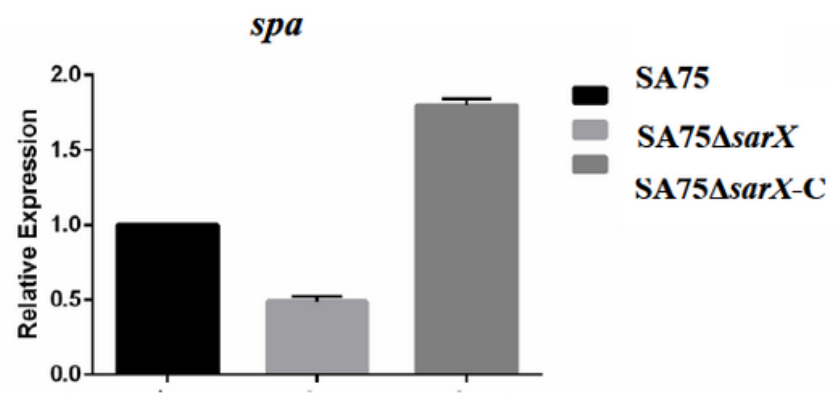

Figure 4

SPA expression levels of SA75, SA75- $\Delta$ sarX, and SA75- $\Delta$ sarX-C RT-PCR results of spa

\section{Supplementary Files}

This is a list of supplementary files associated with this preprint. Click to download.

- supplementPIA.pdf

- supplementPIA.pdf

- supplementSPA.jpg

- supplementSPA.jpg 